\title{
EYE MOVEMENT CONTROLLED PERIPHERALS FOR THE HANDICAPPED-PARALYZED PEOPLE AND ALS PATIENTS
}

\author{
İbrahim Baran USLU ${ }^{1,5, *}$, Fikret ARI ${ }^{2}$, Emre SÜMER ${ }^{3,5}$, Mustafa TÜRKER ${ }^{4,5}$ \\ ${ }^{1}$ Electrical-Electronics Engineering. Department., Engineering Faculty, Atılım University, Ankara, Turkey \\ ${ }^{2}$ Electrical-Electronics Engineering. Department., Engineering Faculty, Ankara University, Ankara, Turkey \\ ${ }^{3}$ Computer Engineering Department., Engineering Faculty, Başkent University, Ankara, Turkey \\ ${ }^{4}$ Geomatics Engineering Department., Engineering Faculty, Hacettepe University, Ankara, Turkey \\ 5 Gözlem; Software, R\&D, and Trade Cooperation, Ankara, Turkey
}

\begin{abstract}
Controlling some devices in their daily life for the handicapped-paralyzed people and ALS (Amyotrophic Lateral Sclerosis) patients is an important challenge. In this study, a wearable system, called SmartEyes, is developed. The system is controlled by the eye movements of the user. With the help of this system, two groups of facilities are provided. The first is: communicating with predefined voiced messages, valuable especially for people who are unable to talk, and the second is: controlling some peripherals which are in the range around the user. The novelty of the developed system is that it navigates among the menus by means of the eye movements with the help of synthesized voice messages and without a need to sit across a monitor. In the control part, both the infrared (IR) and radio frequency (RF) wireless technologies were employed. The details of the peripheral control operations, namely: controlling the desk light, rolling curtain, TV, air conditioner and the sickbed, are explained in detail. The test results show that the system works quite satisfactorily in tracing and implementing the commands given by the user's pupil gaze directions. We found that the overall satisfaction is quite high by yielding a total average survey score of 4.7 out of 5 . We believe that the developed system offers a practical and efficient solution for making the lives of handicapped-paralyzed people and ALS patients easier. We carry on improving the skills of our SmartEyes system.
\end{abstract}

Keywords: Handicapped-paralyzed people, ALS patients, Pupil gaze direction, Wireless hardware control.

\section{INTRODUCTION}

The augmentative technology products helping critical patients, handicapped and paralyzed people are very important. Some of these products offer alternative ways supporting these people for doing their own tasks. An example is the work where one is able to use a computer with the help of the head and eye movements [1]. In another study, some hardware devices in the user's environment can be controlled by head movements which trigger the IR communications with the devices [2].

As is very well known owing to the ice bucket challenge event [3], ALS (Amyotrophic Lateral Sclerosis) is a neurodegenerative disease limiting or blocking people's muscle controlled actions. Although the control over the muscles decreases gradually, the eye muscles usually retain and hence communicating via eyes is mostly available. In [4], after detecting the face by a CCD camera, the defined nine/twelve gaze directions were tried to be identified for selecting the communications choices for ALS patients. In another study [5], the design of a word-processor that works according to the user's finger, eye, and lip motions traced by the sensors, was implemented for the ALS patients. In this work there is also a peripheral control part such as the nurse calling ability.

Eye movement tracking is the technology that has been increasingly used for human computer interaction (HCI) in the last years. This method has also advantages for some usability issues in $\mathrm{HCI}$ 
[6]. There are different techniques for tracking the pupil location in eye tracking. We may group them as the feature-based (eye features) and model-based (searching for the best fitting model) methods. The work of [7] can be given as an example for the first group. The approach in this work uses geometric features of the eyes, such as contours and corners. In a work conducted by [8] an eye camera follows the head movements while maintaining the pupil centred in the image. Such an application was performed as a real-time system for tracking the gaze of a person driving a car. In another study, iris detection was employed for the possible HCI applications, such as wheelchair control or mouse cursor control [9]. One of the most recent works belonging to the first group was conducted by [10], where instead of a monocular; a binocular pupil and gaze detection system was built using LabVIEW. The work of [11] can be given as an example for the second group of eye tracking. In this work, genetic algorithm was adopted.

Most of the previous systems have been intended for only communications or hardware control purposes. The system proposed in this study can both vocalize some pre-defined requests and the needs of the users who are unable to talk, as well as controlling some hardware devices for them. The novelty of the study is that the user does not have to sit across a monitor or some sensors. He/she can use the system at any position, either lying or sitting. The aim is to make these people's lives easier and increase their quality of life.

After this Introduction chapter, in Section-2 we explain the materials and methods, in which the details of the hardware control operations, are given. In Section-3, the results of the preliminary tests are discussed. Finally in Section-4, the conclusion is presented.

\section{MATERIALS AND METHODS}

\subsection{The SmartEyes System}

We developed an eye-controlled wearable system called "SmartEyes" which provides communication and control opportunities for the handicapped-paralyzed people and ALS patients [12]. The system is composed of four modules:

1. Glasses Module: It captures and transmits pupil positions to data processing unit.

2. Interface Module: It configures the system parameters, and associates the pupils with the needs and requests of the patient.

3. Communication Module: It vocalizes the selected needs and requests by navigating on the menu items.

4. Control Module: It controls several household items which are located within the patient's environment.

The SmartEyes system, which was introduced in [12], is extended in this study. The previous system was based on Matlab programming language while the extended version was developed in .NET platform. All image processing algorithms were re-coded using the openCV library. The interface module was re-designed in such a way that the menu items are augmented with the new functions. Some of these functions are represented in Figures 1 and 2. Moreover, a new Graphical User Interface (GUI), which is used to perform the necessary adjustments, was designed (Figure 3). 
Uslu et al. / Anadolu Univ. J. of Sci. and Technology A-Appl. Sci. and Eng. 18 (4) - 2017

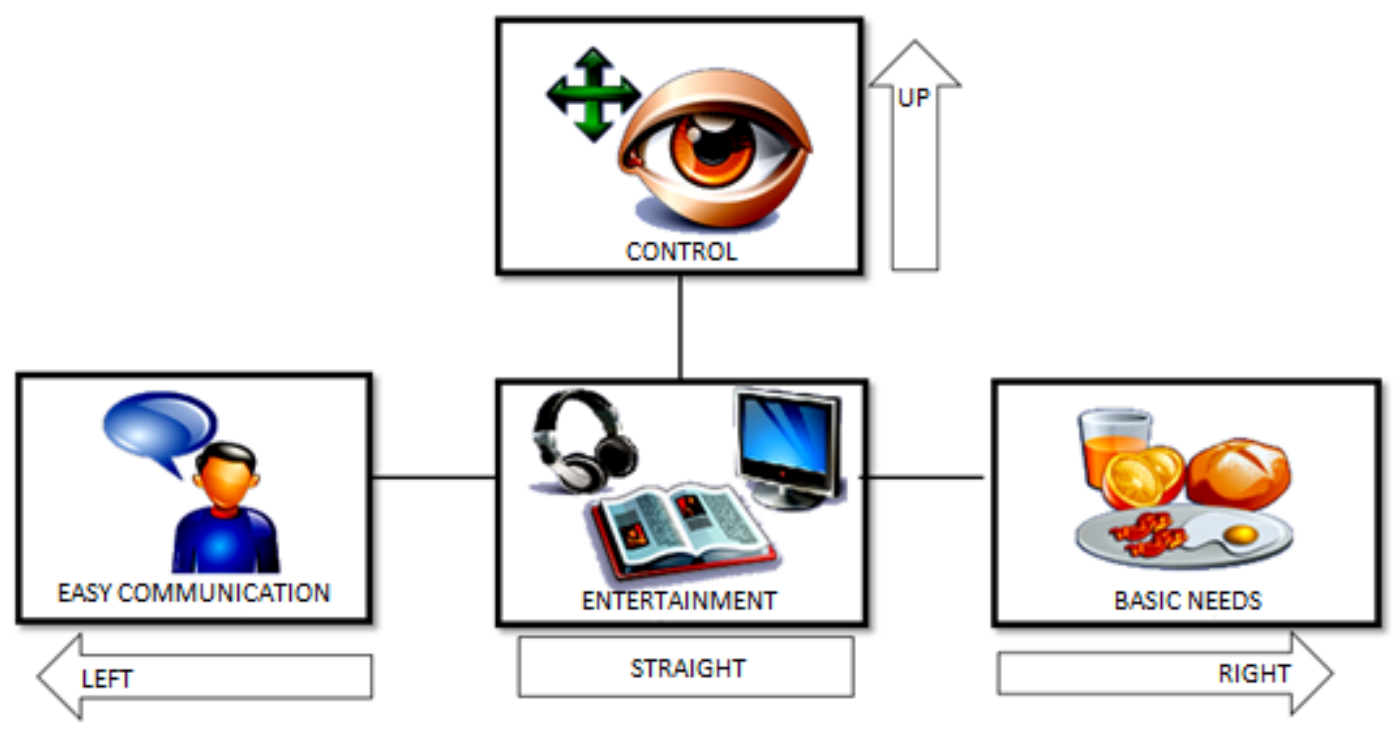

Figure 1. A screenshot of the main menu.



Figure 2. A screenshot of the control menu. 


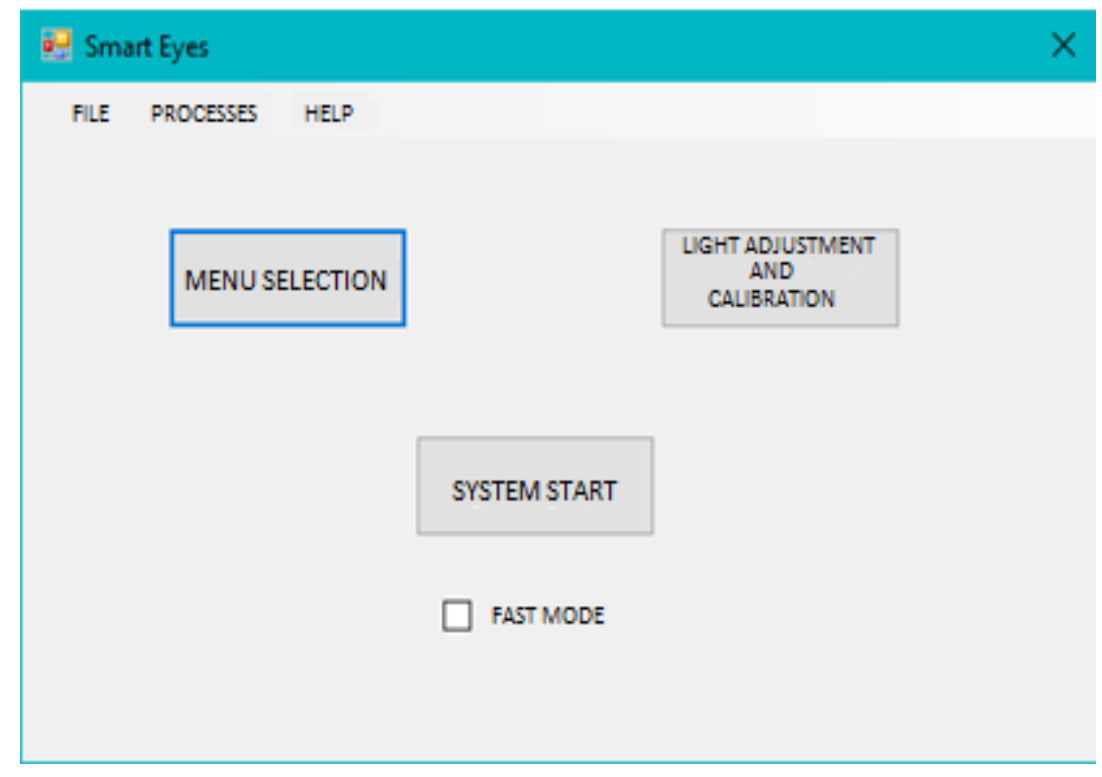

Figure 3. A screenshot of the system adjustment GUI.

In this extended version of the SmartEyes system, the control module was also enhanced by including a "sickbed position adjustment" function. Indeed, this function was highly demanded by the patient surveys, the results of which were published in our previous study [13]. Thus, the peripherals that are controlled by this version of the SmartEyes system are as follows:

- Rolling Curtain

- Desk Lamp

- Television

- Air Conditioner

- Sickbed

The details of each control function are explained in the following sections.

\subsection{Controlling a Rolling Curtain}

In order to control a rolling curtain with SmartEyes, we first acquired an RF remote control rolling curtain (Mosel type) and resolved its RF remote control signals. Then, $433 \mathrm{MHz}$ operating frequency ASK modulated receiver (UDEA ARX-34) was obtained. After that the codes sent by the original remote controller were analyzed with the help of a memory oscilloscope and using a transmitter, (UDEA ATX-34S) which has the same specifications as the receiver, the rolling curtain's control was achieved.

The block diagram of the interface unit designed for the wireless connection of the controller module to the peripherals can be seen in Figure $4 \mathrm{a}$ and its general view in Figure 4b. 
Uslu et al. / Anadolu Univ. J. of Sci. and Technology A-Appl. Sci. and Eng. 18 (4) - 2017
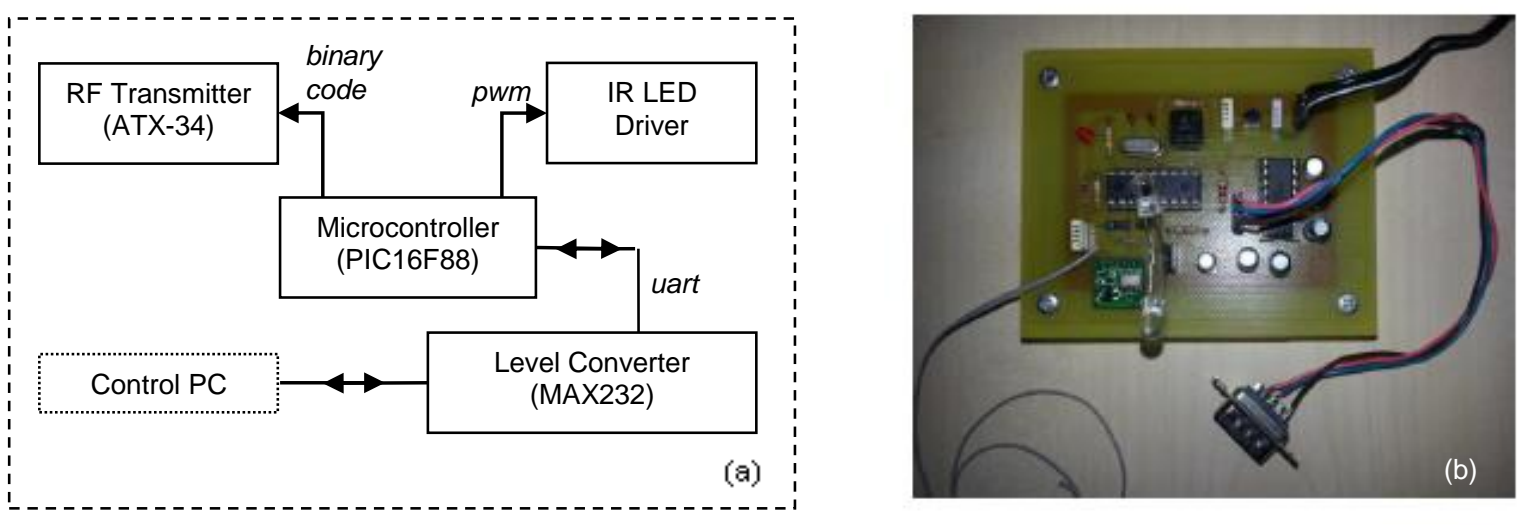

Figure 4. The designed controller card: (a) Its block diagram; (b) Its general view.

The operations that can be performed in controlling a rolling curtain are given in Table 1. In the original controller there were 3 buttons which were for moving the curtain up, stopping it and moving it down. In this study, we added the gradual control motions to the original controller after emulating the original signals and adding timers in between them.

Table 1. The commands for controlling the rolling curtain.

\begin{tabular}{ll}
$\#$ & Operation \\
\hline 1 & Full opening \\
\hline 2 & Full closing \\
\hline 3 & Gradually opening \\
\hline 4 & Gradually closing \\
\hline
\end{tabular}

The oscilloscope screen of a sample RF transmitted signal for the rolling curtain control is also given in Figure 5, where the "roller move up" command is illustrated in detail. Following the "roller move up" command, after a delay time, the "roller stop command" is given. Hence this is the "gradually opening" scenario for the roller curtain.



Figure 5. The RF control signals for gradually opening the rolling curtain. 


\subsection{Controlling a Desk Lamp}

An electronic control unit that contains an IR receiver (TSOP 1136) was designed for controlling a desk lamp. An IR LED transmitter with a wavelength of $940 \mathrm{~nm}$ generates ASK (Amplitude Shift Keying) modulated signals with a $36 \mathrm{kHz}$ carrier wave. Therefore, we used the Philips RC-5 transmission protocol [14] which is based on Manchester coded bit streams. The block diagram and its general view of the designed desk lamp control unit are shown in Figure $6 \mathrm{a}$ and Figure 6b, respectively.
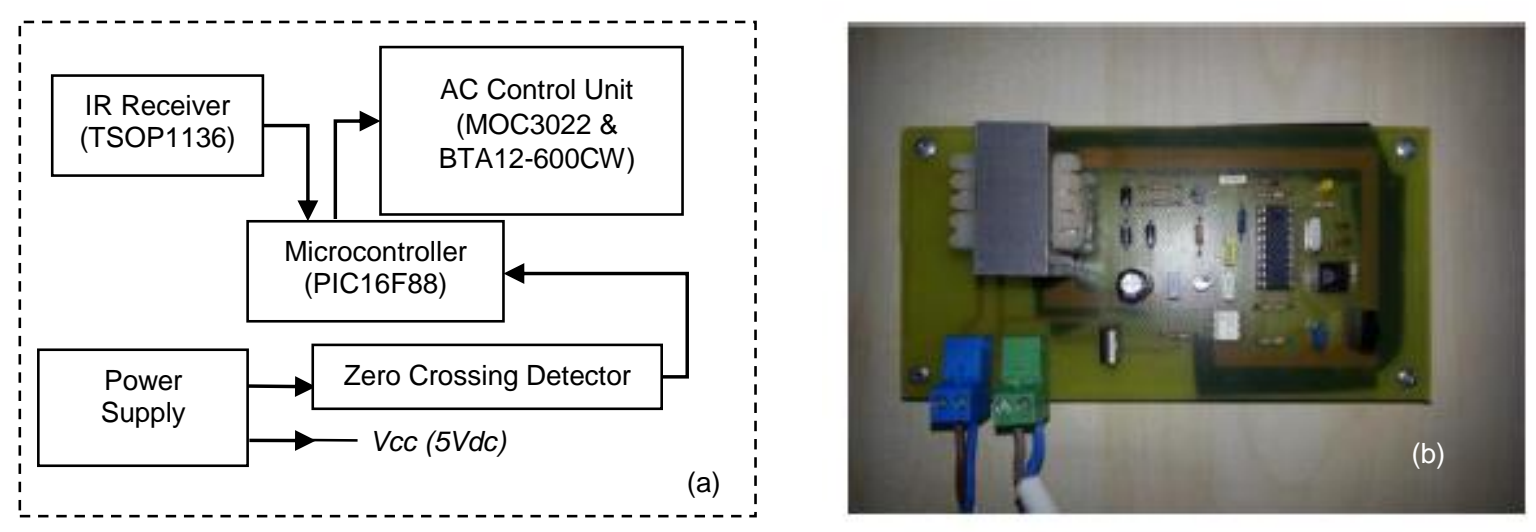

Figure 6. The desk lamp control card: (a) The block diagram; (b) A general view.

Further, dimming the desk lamp can also be controlled by the user. For this purpose, first the zerocrossing points of the mains supply were detected. Then, the dimming was adjusted by means of controlling the phase angle. The details of this control operation are given in Figure 7.

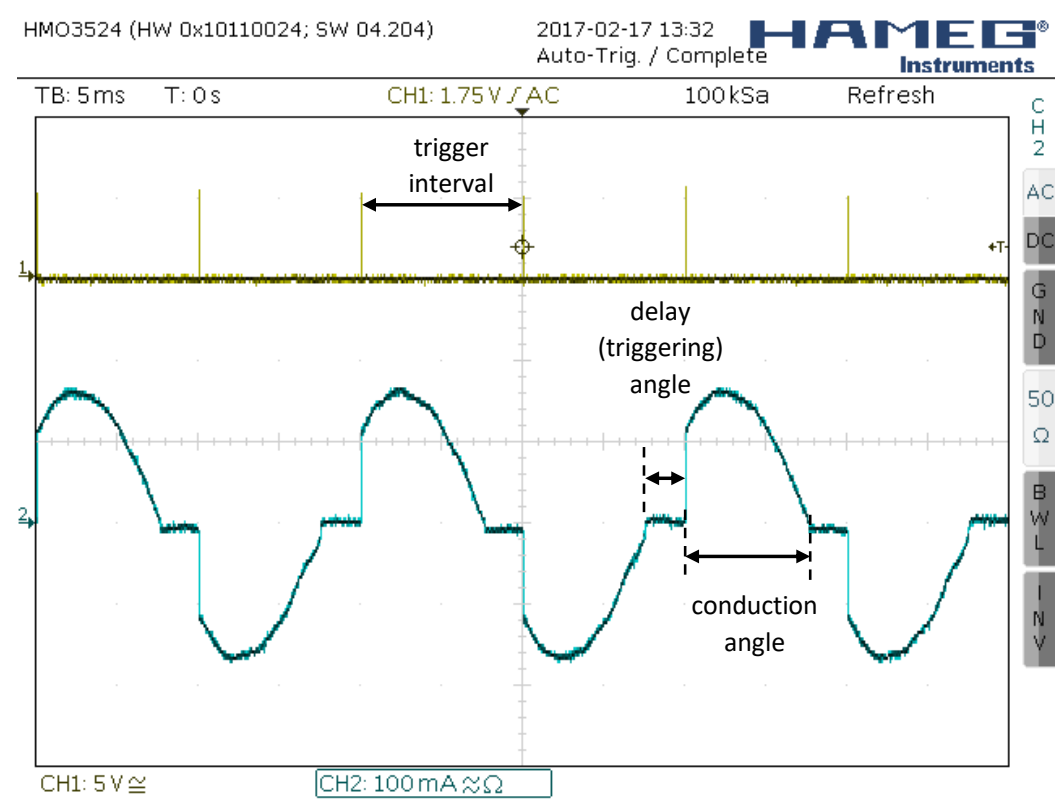

Figure 7. The desk lamp dimming control oscilloscope signals. 
In Figure 7, trace 1 shows the trigger signal's amplitude where each division is $5 \mathrm{~V}$. Each trigger interval is $10 \mathrm{~ms}$ making 2 triggers in the mains period. The duration of each trigger pulse is $10 \mu \mathrm{s}$. These pulses were generated according to the arranged timer interrupt settings. On the other hand, the timer takes the zero-crossings as the reference. By changing the timer settings, the conduction angle can be set between $0^{\circ}$ and $180^{\circ}$. The conduction angle is the interval in which the payload absorbs power from the mains.

Trace 2, in the second channel, shows the phase angle which controls the signal driving the payload by changing the conduction phase angle. It was measured using an AC/DC current probe (ROHDE \& SCHWARZ HZO50). The peak value is near $200 \mathrm{~mA}$ for $220 \mathrm{~V}_{\mathrm{rms}}$ mains supply.

In Figure 7, approximately 70\% efficiency brightness was adjusted.

The operations that can be controlled on the desk lamp are given in Table 2.

Table 2. Commands for controlling the desk lamp

\begin{tabular}{ll}
$\#$ & Operation \\
\hline 1 & Full opening \\
\hline 2 & Full closing \\
\hline 3 & Dimming up \\
\hline 4 & Dimming down \\
\hline
\end{tabular}

\subsection{Controlling a TV}

Similar to the desk lamp controlling approach, the original remote control commands of the selected TV were measured using a TSOP1136 IR receiver and seen that the standard Philips RC5 protocol [14] was used. The codes for the TV control operations, given in Table 3, were generated with the help of the chosen microcontroller (PIC16F88).

Table 3. The implemented TV control commands

\begin{tabular}{ll}
$\#$ & Operation \\
\hline 1 & Turning on \\
\hline 2 & Standby \\
\hline 3 & Channel up \\
\hline 4 & Channel down \\
\hline 5 & Volume up \\
\hline 6 & Volume down \\
\hline 7 & Mute \\
\hline
\end{tabular}

The flowchart of a TV controlling operation (here: channel up) in SmartEyes can also be seen in Figure 8. The user navigates through the menu items with the help of the voiced messages and selects an item between the system's 'Ready' and 'Stop' announcements. 
Uslu et al. / Anadolu Univ. J. of Sci. and Technology A-Appl. Sci. and Eng. 18 (4) - 2017

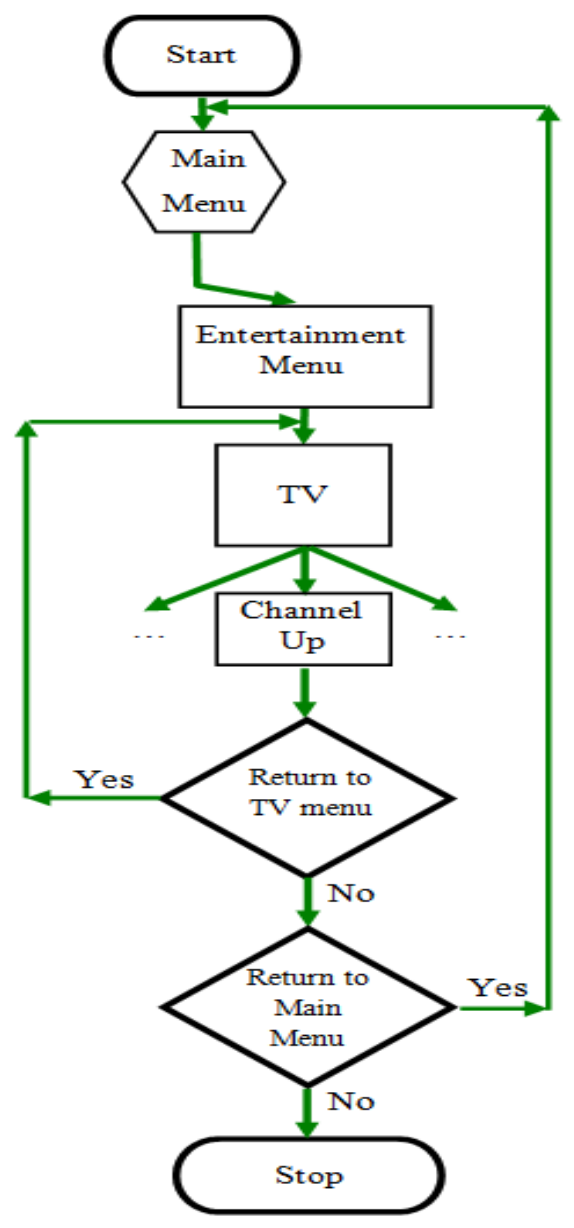

Figure 8. A flowchart of a TV controlling operation (here: channel up) in SmartEyes.

\subsection{Controlling an Air Conditioner (AC)}

For controlling an air conditioner, an IR remote controlled product was acquired and its original commands were resolved using an oscilloscope. Then these commands were defined and added to the software of the designed controller card as shown in Figure 4.

Table 4. The implemented AC control commands

\begin{tabular}{ll}
$\#$ & Operation \\
\hline 1 & Turning on \\
\hline 2 & Standby \\
\hline 3 & Speed up \\
\hline 4 & Speed down \\
\hline 5 & Turn right \\
\hline 6 & Turn left \\
\hline
\end{tabular}

\subsection{Controlling a Sickbed}

A sickbed holds a special position among the peripherals as some handicapped or paralyzed people and some patients have to spend most of their time in a bed. The high rankings in the surveys also support the importance of the sickbed control. 
The available models are mostly wired control; hence it seems that there is a rare need for a wireless control. But just like the other hardware units, so as to make it possible to control the sickbed position, there must be a wireless communications between the SmartEyes system and the sickbed.

The common sickbed models consist of two or three electrical motors for adjusting the head and foot locations. But they do not have a standard communication protocol. Therefore, first we acquired a sickbed and disassembled its motors and the controlling unit. Indeed this process required a precise and dense effort and it is carried out in the laboratory as illustrated in Figure 9.
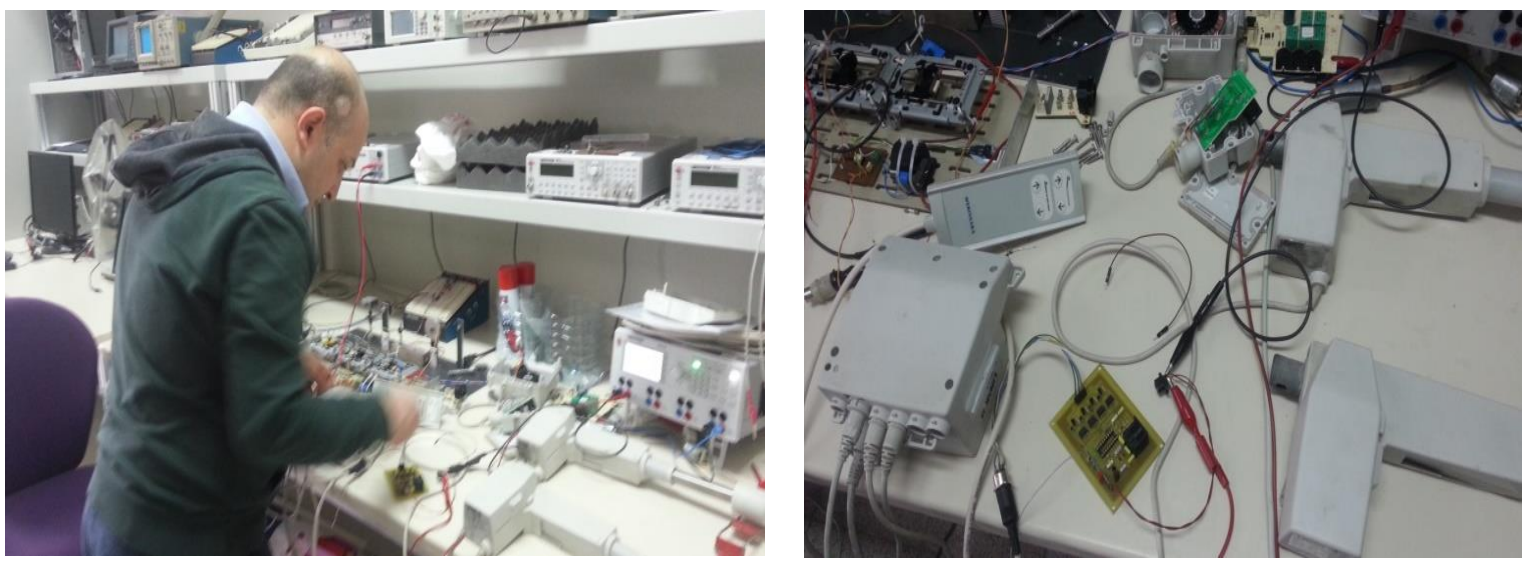

Figure 9. The disassembling process of the motors and the control unit of a sickbed.

A new interface card, whose block diagram can be seen in Figure 10, was designed for the wireless control of the sickbed motors. As can be seen in figure 10, the RF side together with the microcontroller is optically isolated from the sickbed motor driving side for safety purposes. The transistor switching circuit selects from the "head" and "level" motors and its moving direction.

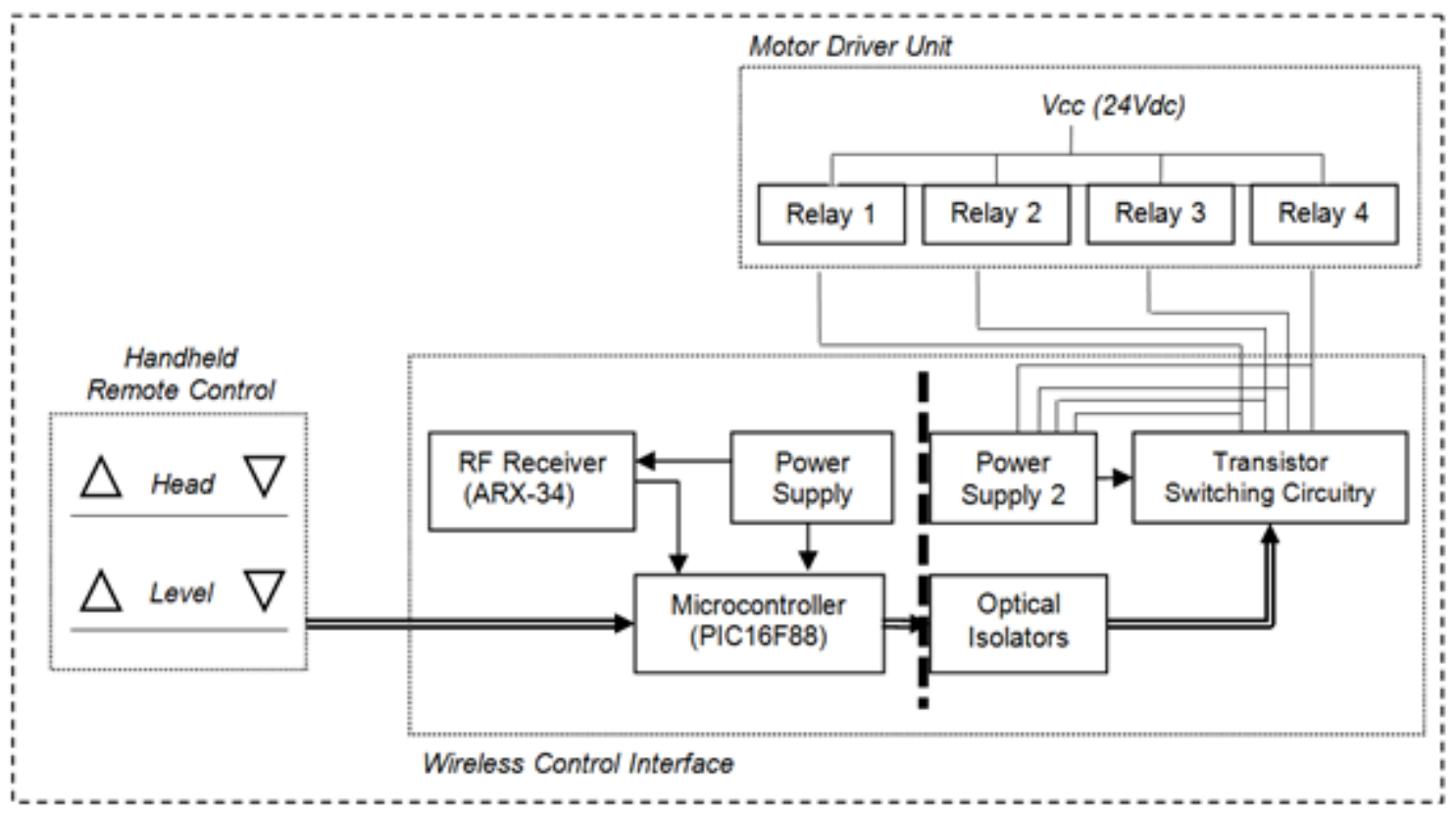

Figure 10. A block diagram of the interface card designed for a wireless sickbed control 
As can be seen in the block diagram shown in Figure 10, there is a microcontroller which traces any signal from both the handheld (wired) controller and the wireless receiver. Therefore, there is no barrier for the caregiver to use the original controller of the sickbed as well. Here again, the UDEA ARX-34, $433 \mathrm{MHz}$ RF receiver was preferred. For the communications protocol, the VirtualWire library, ported from Arduino for PIC microcontrollers [15], was used. The microcontroller then sends the required signals to the motor driving unit. A positive polarity voltage lifts the bed up, while a negative polarity lifts it down. A general view of the designed interface card can be seen in Figure 11.

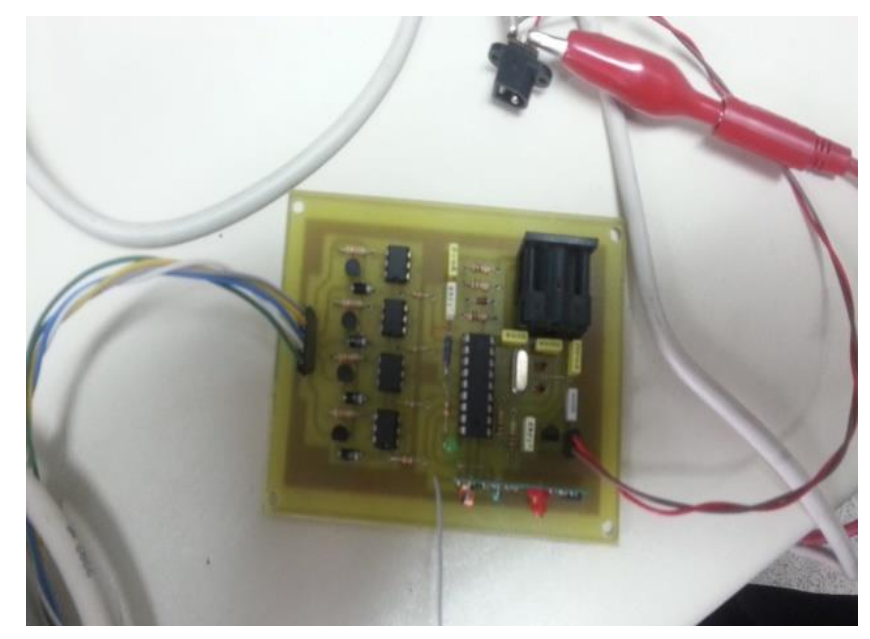

Figure 11. A general view of the interface card designed for the wireless control of a sickbed.

In Figure 12, the controlling signals for the wireless control of the sickbed are shown. Trace 1 is the transmitted bit stream, while trace 2 shows the received signal. As common with many RF modules, there is a preamble part before the payload for synchronizing the transmitter and the receiver.

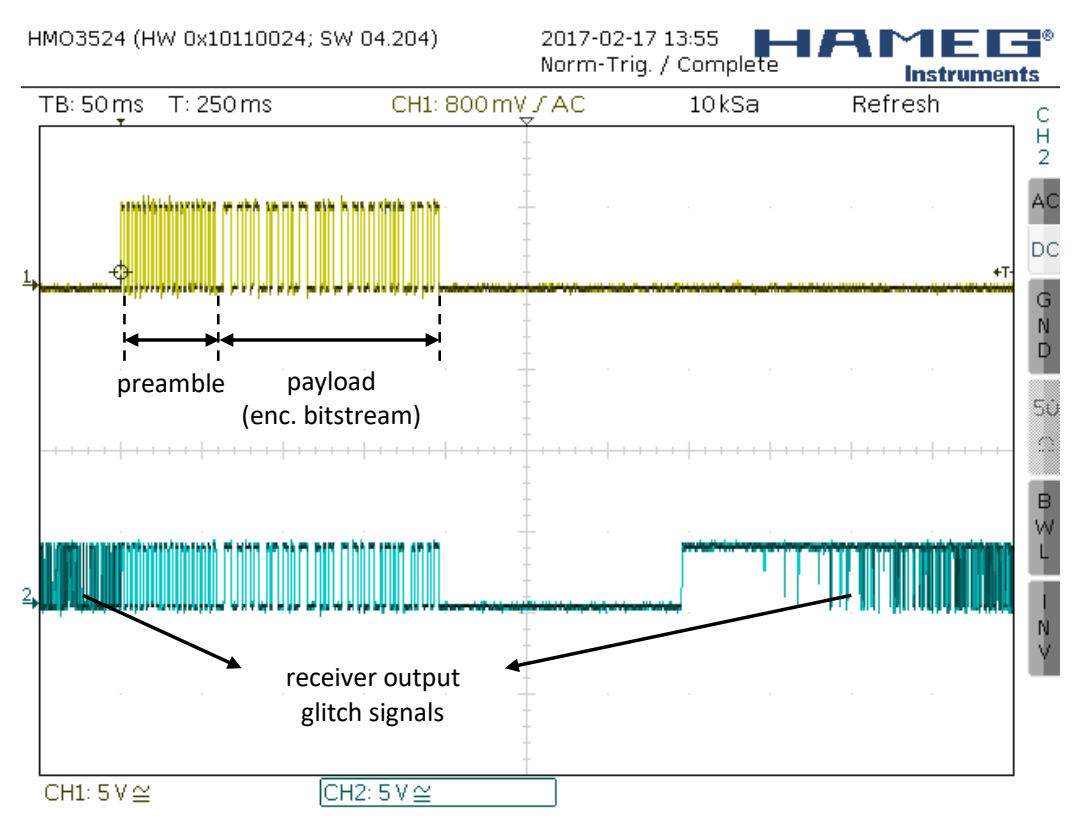

Figure 12. The transmitted and received signals for wireless sickbed control. 
The sickbed control commands are given in Table 5. The user can lift up and down the head and level parts fully and gradually. The commands and their encoded structure are illustrated in Figure 13.

Table 5. Implemented sickbed control commands

\begin{tabular}{llc}
$\#$ & Operation & Command \\
\hline 1 & Full lifting up the head & AHU \\
\hline 2 & Full lifting down the head & AHD \\
\hline 3 & Full lifting up the level & ALU \\
\hline 4 & Full lifting down the level & ALD \\
\hline 5 & Lifting up the head gradually & AHU - AHS \\
\hline 6 & Lifting down the head gradually & AHD - AHS \\
\hline 7 & Lifting up the level gradually & ALU - ALS \\
\hline 8 & Lifting down the level gradually & ALD - ALS \\
\hline
\end{tabular}



Figure 13. The sickbed command structure.

Here, the first character (Unit ID) in the structure indicates the device to be controlled. Alphabetical letters $\mathrm{A}, \mathrm{B}, \ldots$ can be selected for different equipment such as sickbed, wheelchair, etc. The second character (Motor ID) used in the command structure selects basically the subcomponent of the device. As the sickbed used in this study has only two motors to be controlled, the letters $\mathrm{H}$ and L were selected as a reference. The last character (Function) of the command structure indicated as U, D and $\mathrm{S}$ defines the UP, DOWN and STOP controlling functions, respectively.

\section{TEST RESULTS}

The real time tests of the developed SmartEyes system were performed on three groups. In the first group, four company employees tested the prototype. In the second group, healthy people who never used the system before tested the system. There were also four testers with different ages in this group and the tests were carried out in different indoor conditions. Different eye color and the pupil sizes were also the other criteria in selecting the testers. The third and the last group includes two ALS patients. With their permissions, we visited them and they took place in the tests. The views of the tests performed are illustrated in Figures 14 and 15. 

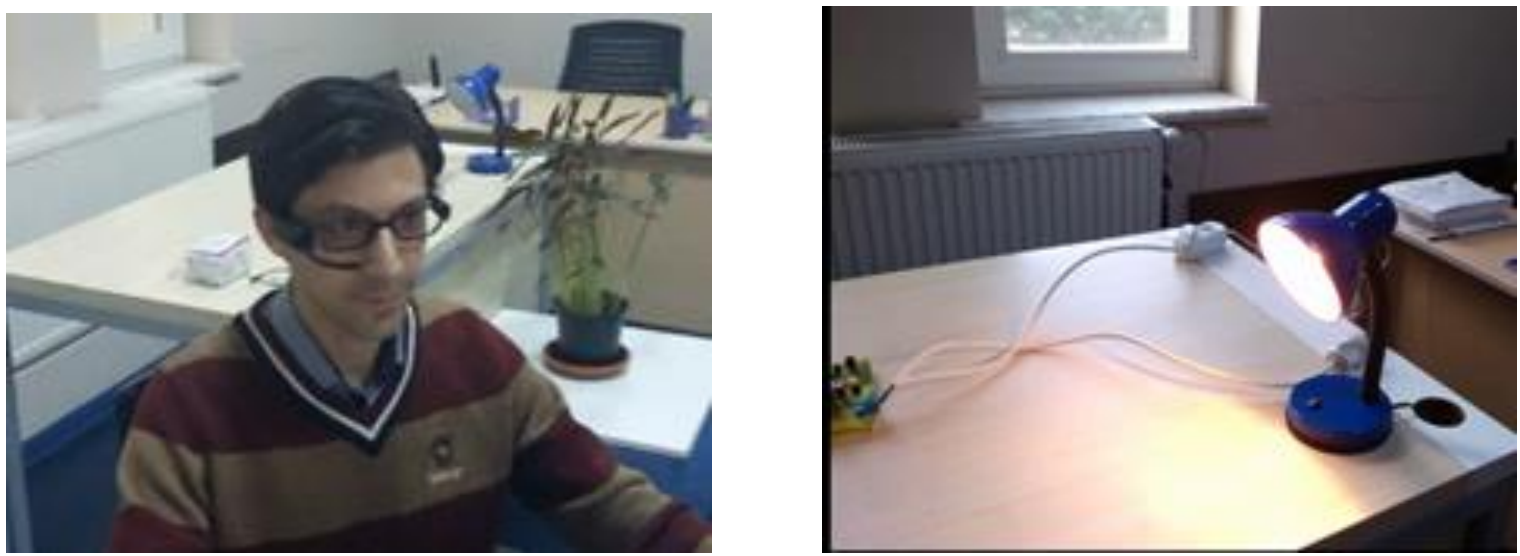

Figure 14. An example test scene (light control)

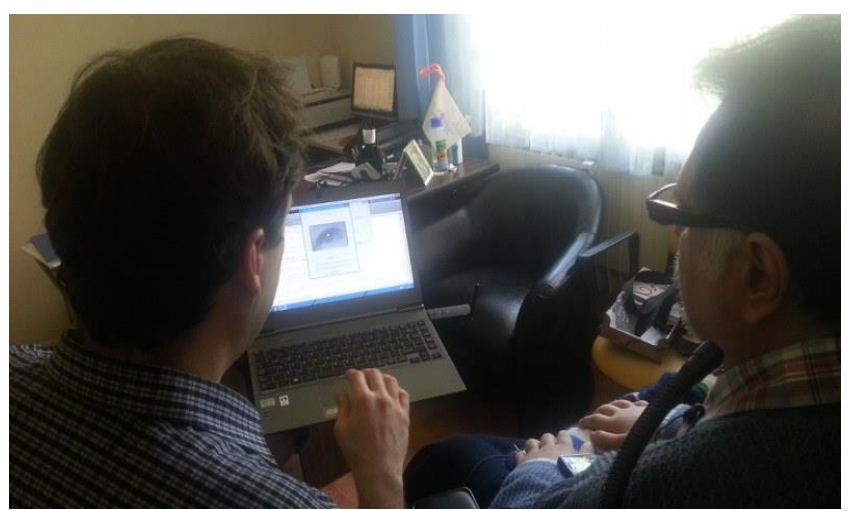

Figure 15. An example test scene (calibration)

The demographic profiles as well as the eye colors of the participants are summarized in Table 6 . The system works consistently for all eye colors, no any critical exceptions are reported. The pupil size changes were also considered. We found that the system works more successfully for those participants who have small and medium pupil sizes. For the large pupil sizes, the system rarely fails due to image segmentation errors. It was also observed that the diameter of the pupil changes by $\% 40$ at most as the source of illumination changes. As expected, the smallest pupil size is obtained in day light while the largest is acquired in dim light.

Table 6. The profile of the participants

\begin{tabular}{ccccc}
\hline Tester ID & Group ID & Age & Gender & Eye Color \\
\hline 1 & 1 & 38 & Male & Hazel \\
\hline 2 & 1 & 41 & Male & Brown \\
\hline 3 & 1 & 27 & Female & Hazel \\
\hline 4 & 1 & 54 & Male & Brown \\
\hline 5 & 2 & 74 & Female & Blue \\
\hline 6 & 2 & 61 & Female & Green \\
\hline 7 & 2 & 37 & Female & Hazel \\
\hline 8 & 2 & 49 & Male & Hazel \\
\hline 9 & 3 & 55 & Male & Brown \\
\hline 10 & 3 & 65 & Male & Brown
\end{tabular}


To assess the usability of the system, a survey was conducted on the participants. In this survey, three questions were asked and for the responses, we used a 5-point Likert scale, in which 1 refers to 'strongly disagree' and 5 refers to 'strongly agree'. The numbers 2, 3 and 4 correspond to 'disagree', 'undecided' and 'agree', respectively. The survey questions that we asked are as follows:

1. How would you assess the functionality of the system?

2. How would you assess the ergonomy of the system?

3. How would you assess the ease of the use of the system?

The survey results are given in Table 7. We found that the overall satisfaction is quite high by yielding a total average score of 4.7 out of 5 . For each question, the average values were computed as $4.8,4.8$, and 4.5 , respectively. It was observed that the participants have used the developed SmartEyes system easily and successfully controlled the dedicated hardware devices.

Table 7. The results of the survey

\begin{tabular}{|c|c|c|c|c|}
\hline Tester ID & Group ID & $\begin{array}{l}\text { Question-1 } \\
\text { Response }\end{array}$ & $\begin{array}{c}\text { Question-2 } \\
\text { Response }\end{array}$ & $\begin{array}{c}\text { Question-3 } \\
\text { Response }\end{array}$ \\
\hline 1 & 1 & 5 & 5 & 5 \\
\hline 2 & 1 & 5 & 5 & 4 \\
\hline 3 & 1 & 5 & 4 & 5 \\
\hline 4 & 1 & 5 & 5 & 4 \\
\hline 5 & 2 & 4 & 5 & 5 \\
\hline 6 & 2 & 5 & 4 & 4 \\
\hline 7 & 2 & 5 & 5 & 5 \\
\hline 8 & 2 & 5 & 5 & 5 \\
\hline 9 & 3 & 4 & 5 & 4 \\
\hline 10 & 3 & 5 & 5 & 4 \\
\hline \multicolumn{2}{|c|}{ Average } & 4,8 & 4.8 & 4.5 \\
\hline \multicolumn{2}{|c|}{ Grand Average } & \multicolumn{3}{|c|}{4.7} \\
\hline
\end{tabular}

\section{CONCLUSION}

In this study, a novel wearable system named SmartEyes was designed and developed. The system provides the ability to navigate between the menu items via the voice messages and allows the user to control some peripherals in a room with the eye movements. The wireless control of the devices, such as rolling curtain, desk lamp, TV, air conditioner and sickbed are now possible as a result of the talent interfacing efforts. One of the main advantages of SmartEyes is that it integrates the communication and control parts in a single system. Besides, by means of voice guidance, the users do not need to look at any screen while using the system. This enables the patients to use the system in any position, such as sitting or in supine position. Furthermore, SmartEyes can operate in dim light due to night vision imaging support. The developed system has several disadvantages. The most important one is the diversity of peripherals to be controlled. For each household item, which does not support remote control, separate receiver circuits should be designed. This may bring a high cost as the number of devices to be controlled increases. Moreover, the infrared control signals may be blocked due to densely furnished indoor environments. In general, we believe that this system will be very useful especially for the handicapped or paralyzed people and ALS patients, providing them the ability to control the households without bothering their caregivers. 
Uslu et al. / Anadolu Univ. J. of Sci. and Technology A-Appl. Sci. and Eng. 18 (4) - 2017

\section{ACKNOWLEDGEMENTS}

The study was supported by TÜBITAK TEYDEB-1512 program with Grant number 2120141 and KOSGEB R\&D and Innovation program.

The authors would like to thank Dr. Alper Kaya (executive board member of Alliance of ALS/MND Associations) for his support and encouragement throughout the SmartEyes project.

\section{REFERENCES}

[1] SmartNav (www.naturalpoint.com - last visit: 29.09.2017)

[2] Nakashima H, et al. Vision device to support independent life for people with serious disability. Multimedia Technology (ICMT), 2011 International Conference on. IEEE, 2011. p. 6160-6163.

[3] Ice Bucket Challenge (http://www.alsa.org/fight-als/ice-bucket-challenge.html -last visit: 29.09.2017)

[4] Maehara T, et al. A communication system for ALS patients using eye-direction. IEEE EMBS Asian-Pacific Conference on. IEEE, 2003. p. 274-275.

[5] Yamada M and Fukuda T. Eye word processor (EWP) and peripheral controller for the ALS patient. IEE Proceedings A (Physical Science, Measurement and Instrumentation, Management and Education, Reviews), 1987, 134.4: 328-330.

[6] Poole A and Ball L J. Eye tracking in HCI and usability research. Encyclopedia of human computer interaction, 2006, 1: 211-219.

[7] Cuong $\mathrm{N} \mathrm{H}$ and Hoang $\mathrm{H} \mathrm{T}$. Eye-gaze detection with a single WebCAM based on geometry features extraction. Control Automation Robotics \& Vision (ICARCV), 2010 11th International Conference on. IEEE, 2010. p. 2507-2512.

[8] Pérez A, Córdoba M L, García A, Méndez R, Muñoz M L, Pedraza J L, Sánchez F A . Precise eyegaze detection and tracking system. WSCG'2003, February 3-7, 2003, Plzen, Czech Republic.

[9] Pathak Y, Akhare S, Lambe V. Iris movement tracking by morphological operations for direction control. International Journal of Engineering Research \& Technology (IJERT) Vol. 1 Issue 7, September - 2012.

[10] Durna Y and Arı F. Design of a binocular pupil and gaze point detection system utilizing high definition images. Applied Sciences, 2017, 7.5: 498.

[11] Akashi T, et al. Using genetic algorithm for eye detection and tracking in video sequence. Journal of Systemics, Cybernetics and Informatics, 2007, 5.2: 72-78.

[12] Sümer E, Uslu B, Türker M. An eye-controlled wearable communication and control system for ALS patients: SmartEyes. Sigma J. Eng \& Natural Sciences 2017; 8(2): 107-116.

[13] Uslu İ.B., Sümer E., Türker M. and Arı F. ALS hastaları için göz bebeği hareketlerine dayalı çevre birimi kontrolü (in Turkish). Otomatik Kontrol Ulusal Toplantısı (TOK-2016), Osmangazi Üniversitesi, Eskişehir, 29.09 - 01.10.2016.

[14] Remote Control System RC-5 Including Command Tables, Philips Semiconductors, December 1992, Publication No. 938870623011

[15] Arduino VirtualWire for PIC Controllers (http://www.enide.net/webcms/?page=virtualwire-forpic - last visit: 29.09.2017) 